\title{
EVALUATION OF HEMOGRAM AND BIOCHEMICAL ANALYSIS OF EQUINE CLINICALLY INFECTED WITH BABESIOSIS
}

K.M. ALSAAD* and DHIYAA A. MUSSA

*E.mail:kamalsad58@yahoo.com

\section{ABSTRACT}

Received: 30/5/2012

\section{Accepted at:}

Hemogram, acid base balance, blood gas analysis and acute phase response have been evaluated in horses clinically infected with babesiosis in Mosul, Iraq. The study was conducted on 100 local horse breed ranging from 3-10 years old of both sexes. Eighty local horse breed were clinically infected with Babesia spp.which diagnosed by Giemsa stained blood smears and confirmed by C-Elisa test. 20 clinically normal horses served as control group. Results indicated statistically significant decrease $(\mathrm{P}<0.05)$ in $\mathrm{TRBCs}, \mathrm{Hb}$, and $\mathrm{PCV}$ values in diseased animals, Macrocytic hypochromic type of anemia was found and the percentage of hemoparasitism (parasitemia) ranged between $(5-24 \%)$ with a mean of $(16.57 \%)$. The results also indicated a significant increase in TLC as a result of significant increase of lymphocytes. Babesia equi were detected in $63.75 \%$ whereas Babesia caballi detected in $36.25 \%$. Statistically significant decrease were encountered in blood $\mathrm{pH}, \mathrm{PCO}_{2}$, bicarbonate ions, Base excess and Oxygen saturation percent $\left(\mathrm{So}_{2}\right)$ in diseased horses than in controls, However statistically increase in anionic gab have been detected, Moreover titrational metabolic acidosis were indicated. Statistically significant difference have been encountered in acute phase response as haptoglobin and fibrinogen values were decreased in clinically infected horses. It have been concluded that different adverse effect, hemolytic anemia, tissue hypoxia beside decrease acute phase response were encountered in horses infected with Babesia spp.

\section{Key words:}

\section{التقييم الاموي والتحليل البيوكيميائي للخيول المصابة سريريا بداء الكمثريات}

\section{كمال الدين مهيل السعد ، ضياء عبل الله موسى}

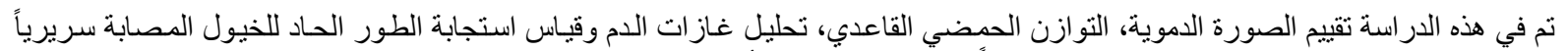

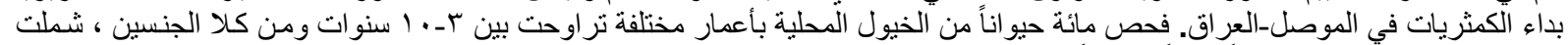

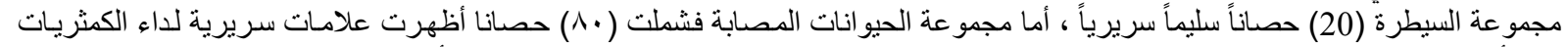

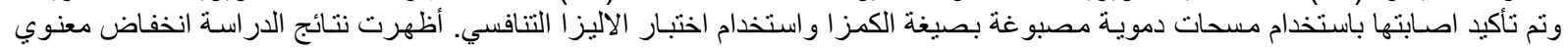

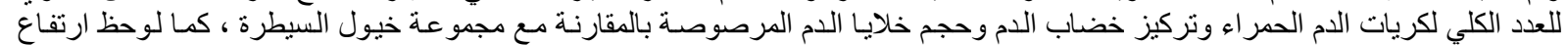

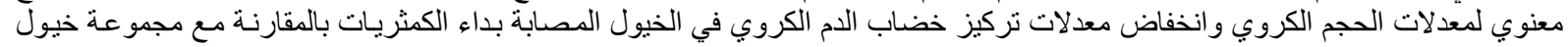

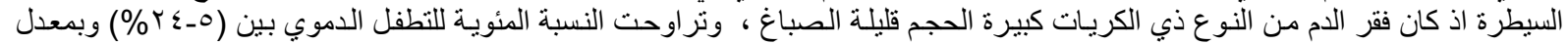

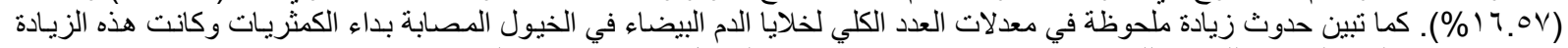

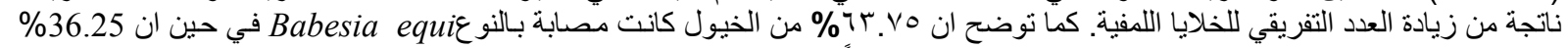

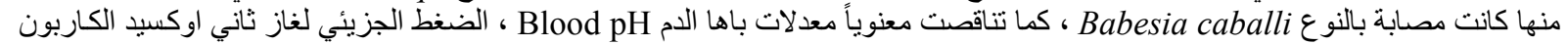

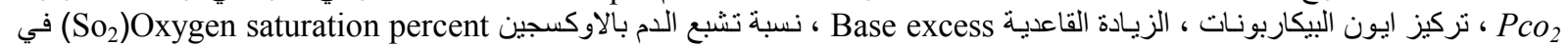

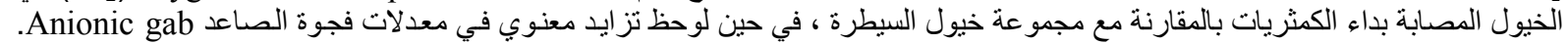

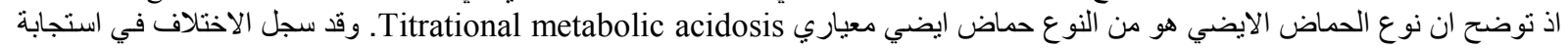

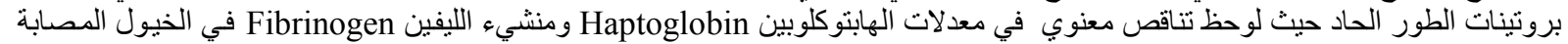

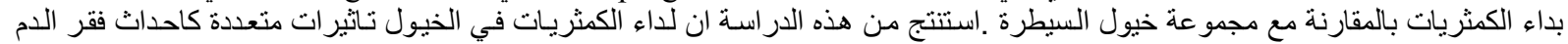

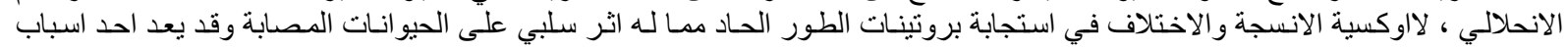




\section{INTRODUCTION \\ المقدمسـة}

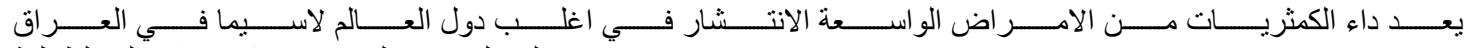

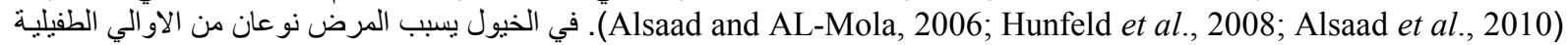

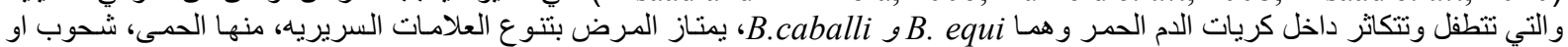

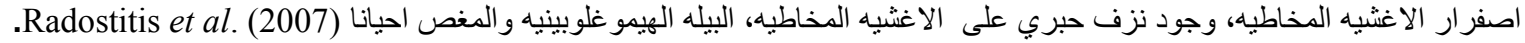

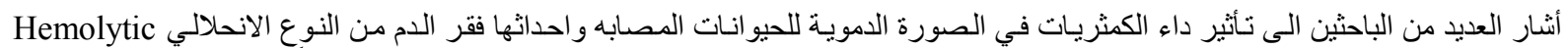
anemia Zambelli, ) فضـلا عن اضطر اب التوازن الحمضي_ القاعدي (Zobba et al., 2008 and Alsaad et al., 2010) Tissues hypoxia 2007). يمكن تقييم معاملات التوازن الحمضي القاعدي و اكسجة Oxygenation الدام (قياس مستوى الضغط الجزئئي للاوكسجين

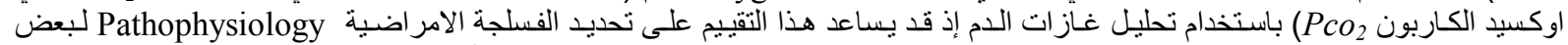

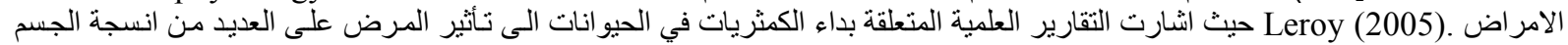

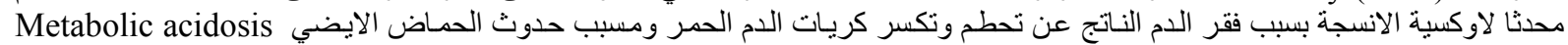

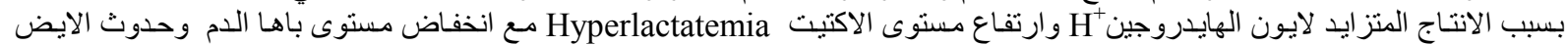

اللاهو ائي الانتاج (Welzl, 2001; Lobetti et al., 2002; Vannier and Krause, 2009) Anaerobic mtabolism)

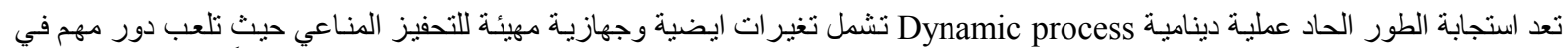

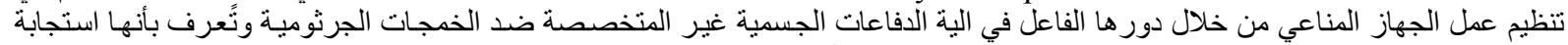

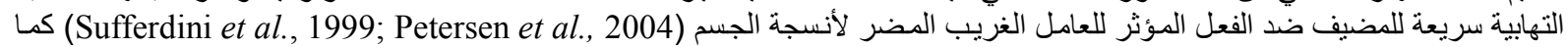

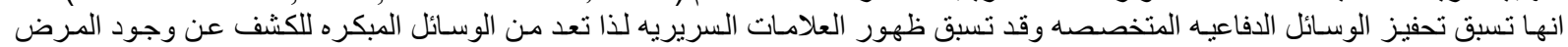

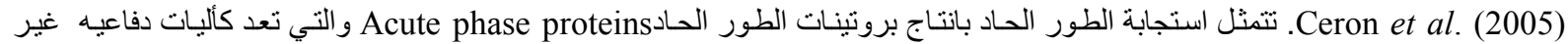

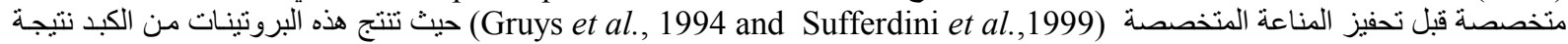

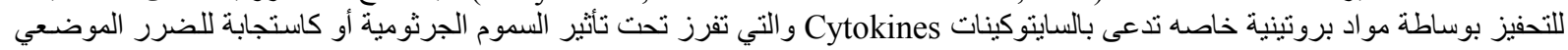

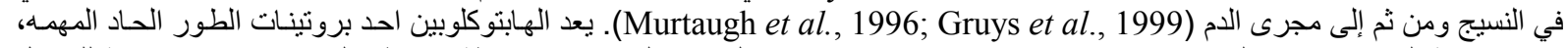

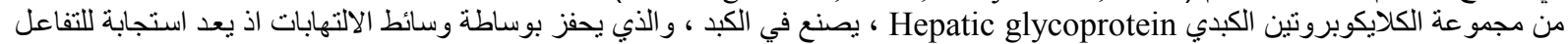

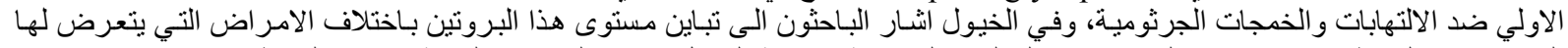

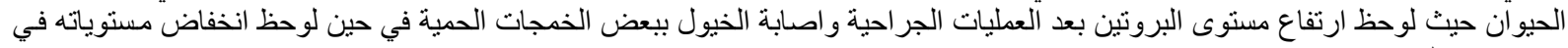

حالات فقر الدم (Hulten et al., 1999; Hulten et al., 2002).

يهدف البحث الى در اسة ناثير داء الكمثريات على الصورة الدموية فضلا عن تقييم التوازن الحمضي القاعدي، تحليل غاز ات الدم وتقييم استجابة

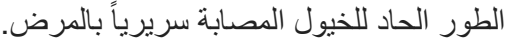

\section{MATERIALS and METHODS}

المواد وطرق البحث

اولاًا: حيوانات الاراسة

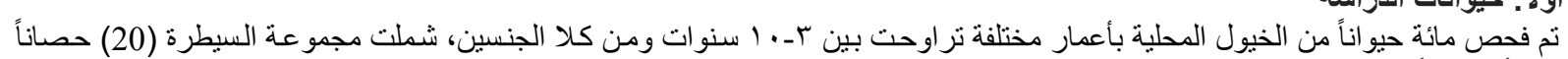

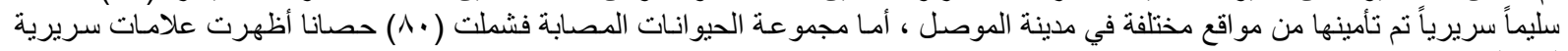
لداء الكمثريات.

ثانياً: الفحوصات المختبرية

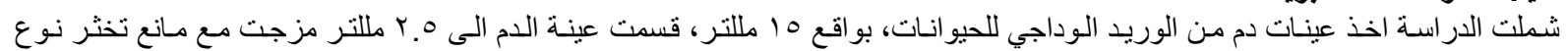

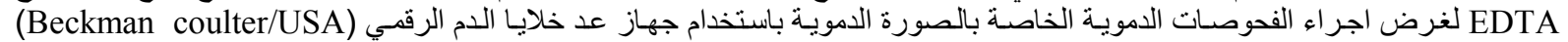

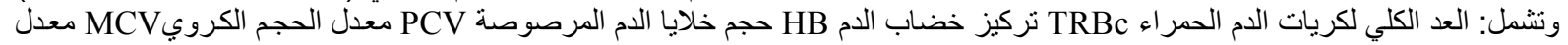

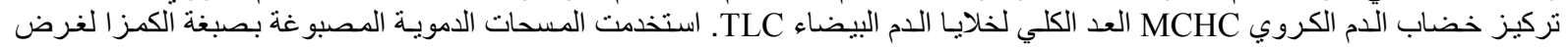

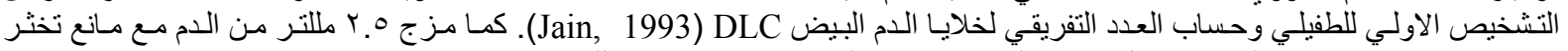

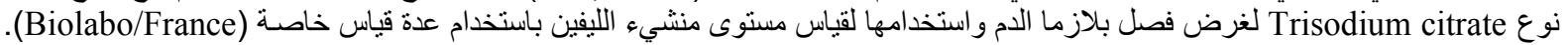

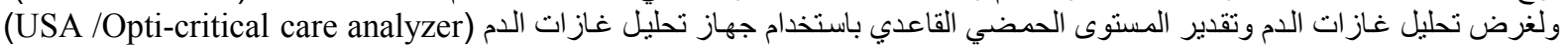

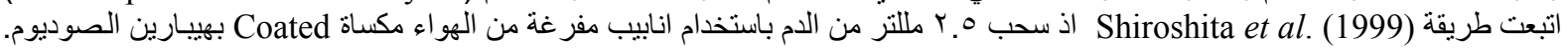

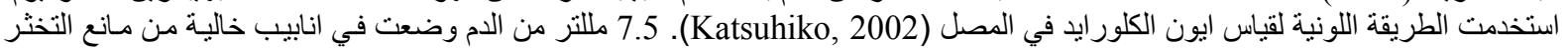

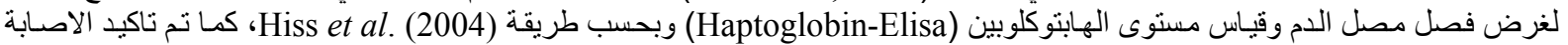

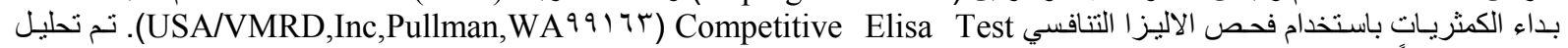

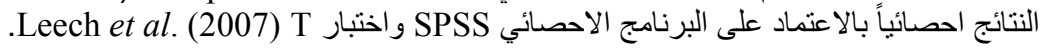

\section{RESULTS \\ النتائسج}

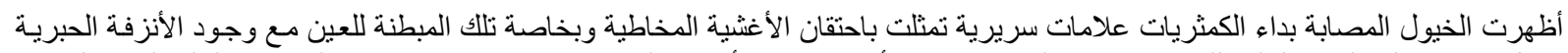

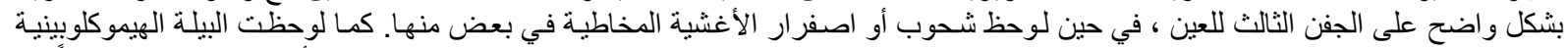

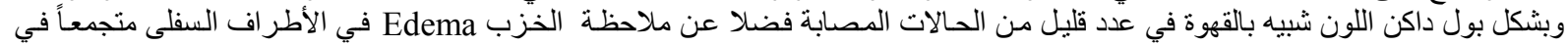

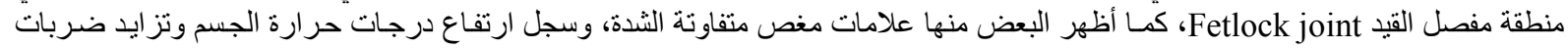
القلب وترداد التنفس في جميع الخيول المصابة. 


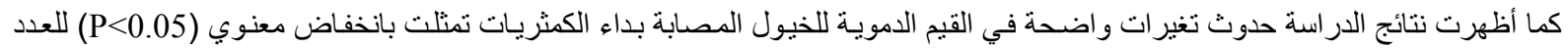

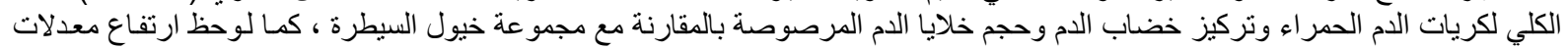

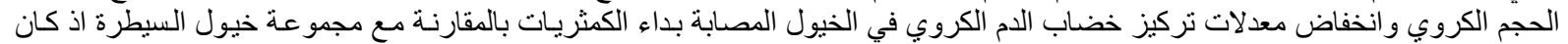

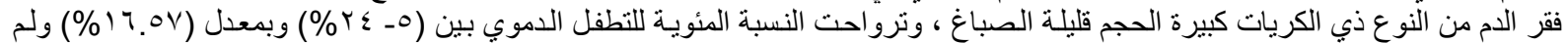
يلاحظ الطفيلي بكلا نو عيه في المسحات الدموية لمجمو عة خيول السيطرة، جدول ( ( ).

جدول 1: التغيرات في المعايير الدموية في الخيول المصابة بداء الكمثريات ومجموعة خيول السيطرة

\begin{tabular}{|c|c|c|}
\hline \multicolumn{2}{|c|}{ المعدل 土 الخطأ القياسي } & \multirow{2}{*}{ المعـــــير } \\
\hline الخيول المصابة & مجمو عة السيطرة & \\
\hline$\because \varepsilon V \pm V . Y T$ & $.90 \pm 9.91$ & العدد الكلي لكريات الدم الحمر (x ’ ، مايكروليتر) \\
\hline$. . M \pm \wedge . M r$ & $\cdot .7 \pm 1 \Upsilon . \wedge \mathrm{V}$ & تركيز خضاب الدم ( غرام / . . ( مللتر ) \\
\hline $1.50 \pm r V .70$ & $1.10 \pm r \leqslant . Y 7$ & حجم خلايا الدم المرصوصة ( \% ) \\
\hline$T . \leqslant \neg \pm r \wedge . Y \leqslant$ & $1.0 V \pm r \leqslant .0 V$ & معدل الحجم الكروي ( فيمتوليتز ) \\
\hline $0.0 V \pm Y \Lambda . Y I$ & $\cdot . \leqslant 0 \pm \varepsilon \cdot . \leqslant \Lambda$ & معدل تركيز خضاب الدم الكروي ( غر ام/ + . مللتر) \\
\hline$\varepsilon . \wedge 1 T_{ \pm 117.0 V}$ & ------------ & نسبة التطفل الدموي \% \\
\hline
\end{tabular}

(P<0.05) مستوى المعنوية * مبنه

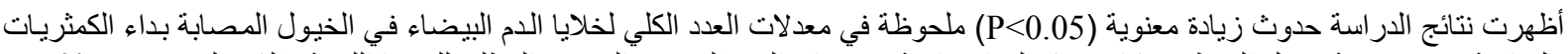

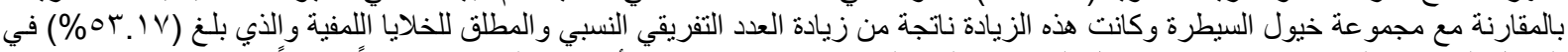

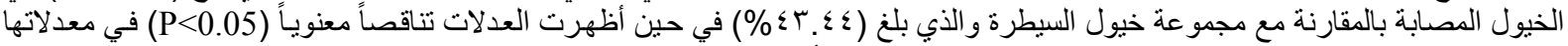

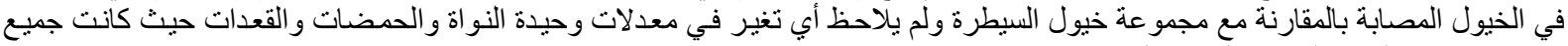

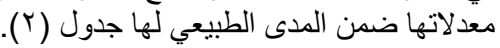

جدول r : العدد الكلي و التفريقي (النسبي و المطلق) لخلايا الدم البيضاء في الخيول المصابة بداء الكمريات ومجموعة خيول السيطرة المعدل 土 الخطأ القياسي

\begin{tabular}{|c|c|c|c|}
\hline الخيول المصابة & مجمو عة السيطرة & & \\
\hline$\left.{ }^{*}\right) . \wedge \cdot \pm 1 r .1 \wedge$ & $\cdot r \leq \pm 1 \cdot r_{0}$ & x' • · مايكروليتر ) & العدد الكلي لخلاي \\
\hline${ }^{* T . Y V} \pm 0 \mathrm{Or} . \mathrm{IV}$ & $Y . V V \pm \varepsilon r . \varepsilon \varepsilon$ & $(\%)$ & \multirow{2}{*}{ الخلايا اللمفيـــة } \\
\hline *Vนr.r & $100.1 \pm \leqslant \leqslant 0 Y .7$ & مطلق & \\
\hline$* \varepsilon . Y \neg \pm r V . \wedge I$ & $r .9 \wedge \pm \leqslant \vee .1 \leqslant$ & $(\%)$ & \multirow{2}{*}{ العــــلات } \\
\hline *ร $\leqslant \varepsilon .99 \pm \varepsilon q \wedge r . r$ & $107.1 \varepsilon_{ \pm} \leqslant \Lambda \mu 1 . \wedge$ & مطلق & \\
\hline $1.07 \pm \varepsilon .1 \varepsilon$ & $Y, Y Y \pm \varepsilon . Y I$ & $(\%)$ & \multirow{2}{*}{ وحيدة النــــواة } \\
\hline $1.0 .91 \pm 0 V Y$ & $\mid r T .0 \wedge \pm \varepsilon T 1.0 T$ & مطلق & \\
\hline $1 . V Y_{ \pm} 0 . \wedge V$ & $.0 \leq \pm 5.11$ & $(\%)$ & \multirow{2}{*}{ الحمضــــات } \\
\hline $107 . \Lambda \Lambda \pm 0 V r . r$ & $r \cdot \wedge . \vee \wedge \pm O r r . V V$ & مطلق & \\
\hline$\cdot . T \pm \pm \cdot V T$ & $\because \vee V 7 \pm \cdot . V 1$ & $(\%)$ & \multirow{2}{*}{ القعـــــــات } \\
\hline$\wedge \circ . r \pm 76$ & $V \wedge . Y Y \pm V Y . O Y$ & مطلق & \\
\hline
\end{tabular}

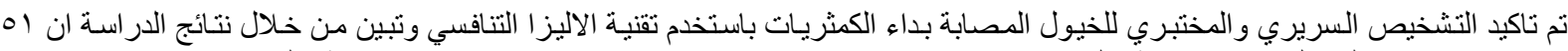

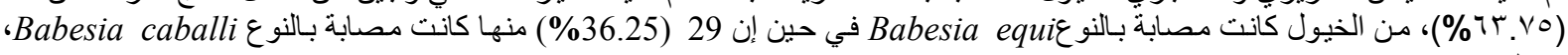

جدول (r) (T)

جدول ب: نتائج اختبار الاليزا التنافسي في الخيول المصابة بداء الكمثريات

\begin{tabular}{|c|c|c|c|c|}
\hline المجموع & العينات السالبه مصليا & العينات الموجبه مصليا & اختبار الاليز ا التنافسي لطفيلي & $ت$ \\
\hline$\wedge$. & $(\% 36.25) 29$ & $(\% 7 r . \vee 0) 01$ & Babesia equi & 1 \\
\hline A. & $(\% 7 r . v 0) 01$ & $(\% 36.25) 29$ & Babesia caballi & $r$ \\
\hline
\end{tabular}




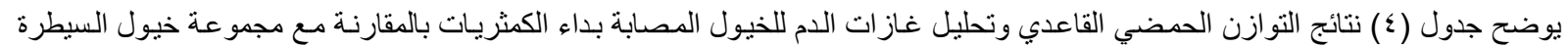

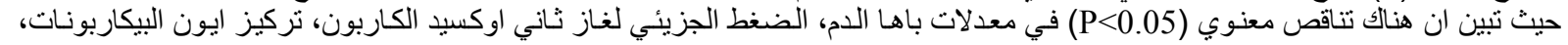

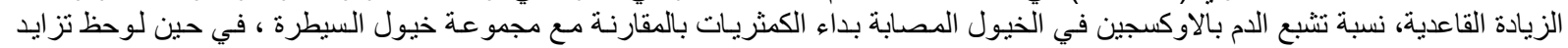

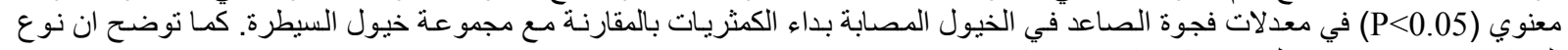

الحماض الايضي هو من النوع حماض ايضي معياري Titrational metabolic acidosis.

جدول ؛ : نتائج التو ازن الحمضي- القاعدي وتحليل غازات الدم في الخيول المصابه بداء الكمثريات ومجمو عة خيول السيطرة

\begin{tabular}{|c|c|c|}
\hline \multicolumn{2}{|c|}{ المعدل \pm الخطأ القياسي } & \multirow{2}{*}{ المعــــير } \\
\hline الخيول الدصابة & مجمو عة السيطرة & \\
\hline${ }^{*} . .7 Y \pm 7.90$ & $\because Y Y \pm . \varepsilon r$ & باها الدم \\
\hline$* r .09 \pm\{\cdot . Y)$ & $1.70 \pm \leqslant 0.91$ & الضغط الجزيئي لغاز ثاني اوكسيد الكاربون ( ملم زئبق) \\
\hline${ }^{* 1} .7 r \pm 19.74$ & $r . \wedge 9 \pm Y \varepsilon . r \wedge$ & تركيز ايون البيكاربونيت (ملي مول/ لتر) \\
\hline $0 . Y Y \pm 101.7 V$ & $\left.\varepsilon . r r_{ \pm}\right) \leqslant q$ & الضغط الجزيئي لغاز الاوكسجين (ملم زئبق) \\
\hline${ }^{*} 0.48 \pm(-5.95)$ & $1.12 \pm 4.74$ & الزياده القاعديه (ملي مول / لتر) \\
\hline${ }^{*} Y .07 \pm I Y . Y Y^{2}$ & $1.0 \leqslant \pm 1.11$ & فجوة الصاعد (ملي مول / لتر) \\
\hline$* \%$ \%० & $\% 9 \Lambda$ & نسبة تشبع الدم بالآوكسجين \% \\
\hline$\varepsilon .17 \pm 1 \mu_{0}$ & $r . r \theta_{ \pm} / \mu \Lambda$ & تركيز ايون الصوديوم (ملي مول/ لتر) \\
\hline$\because \vee \wedge \pm r .79$ & $\because V 0_{ \pm} r .71$ & تركيز ايون البوتاسيوم (ملي مول/ لتر) \\
\hline$\varepsilon .1 \vee \pm 91.70$ & I. $r \Psi \pm 99 . \wedge r$ & تركيز ايون الكلور ايد (ملي مول/ لتر) \\
\hline
\end{tabular}

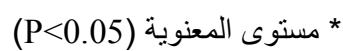

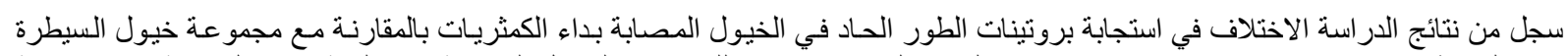

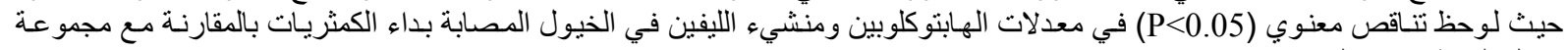

خيول السيطرة. جدول(0) (0)

جدول ه: مستويات بروتينات الطور الحاد (منشئ الليفين والهابتوكلوبين) في الخيول المصابة بداء الكثريات ومجموعة خيول السيطرة

\begin{tabular}{|c|c|c|}
\hline \multicolumn{2}{|c|}{ المعدل 土 الخطأ القياسي } & \multirow{2}{*}{ المعــــير } \\
\hline الخيول المصابة & مجموعة السيطرة & \\
\hline${ }^{*} \cdot . \cdot 0 \pm Y . \varepsilon \cdot$ & $\because \cdot 0 \pm r . \cdot v$ & منشئ الليفين ( غرام / . ( ملبلتر ) \\
\hline$* \cdot \cdot \varepsilon \pm \cdot V 1$ &.$\| r \pm r . r r$ & الهابتو كلو بين (ملغم / . . 1 مليلتر ) \\
\hline
\end{tabular}

(P<0.05) مستوى المنوية *

\section{DISCUSSION}

\section{المناقثة}

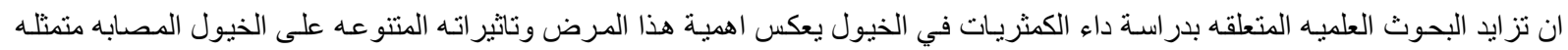

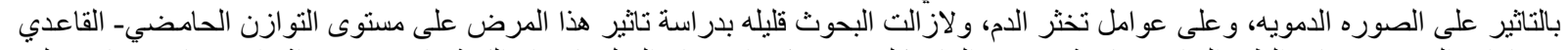

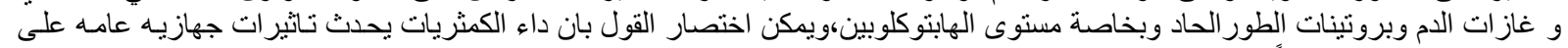

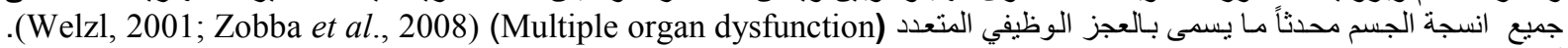

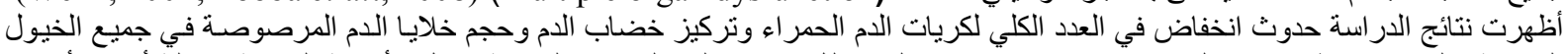

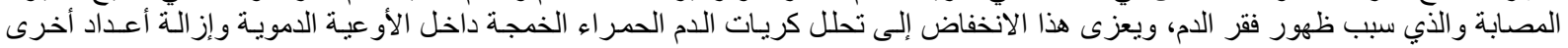

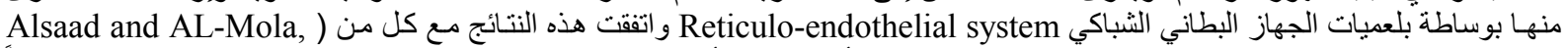
2006; Zobba et al.,2008; Alsaad et al., 2010

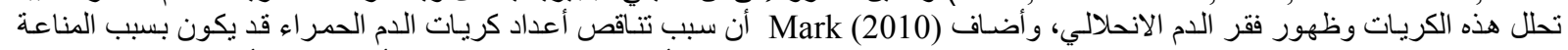

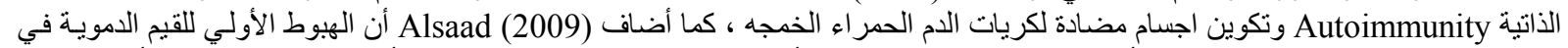

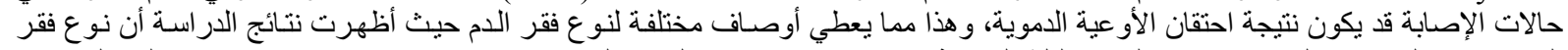

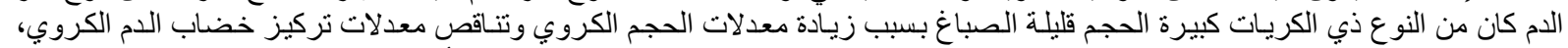

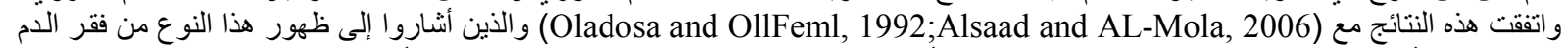

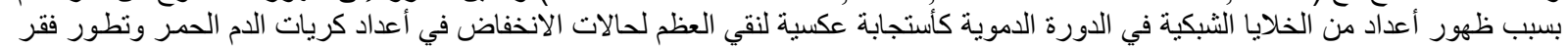

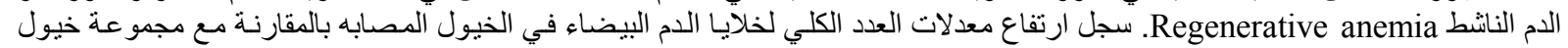

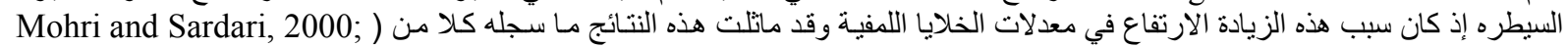

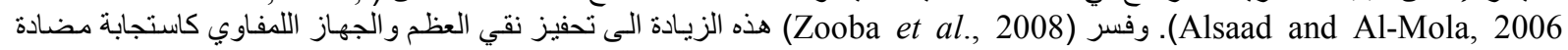

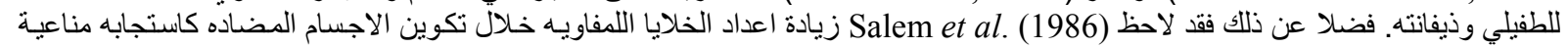

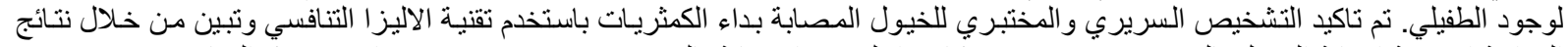

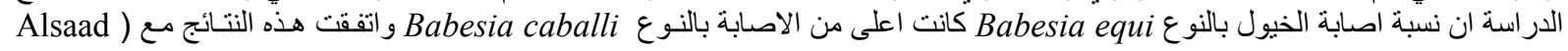




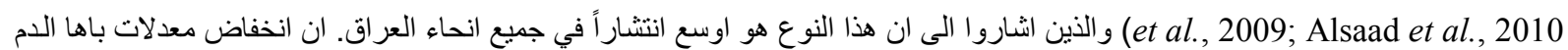

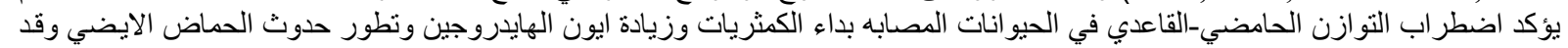

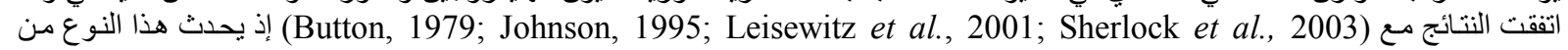

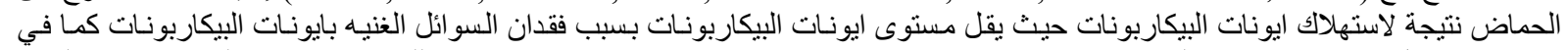

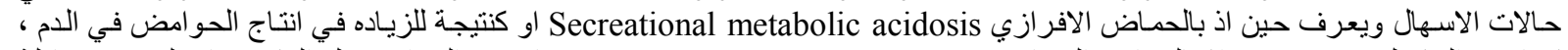

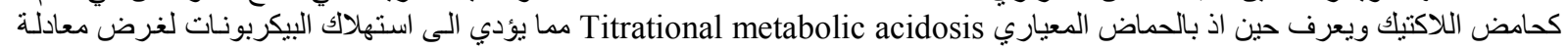

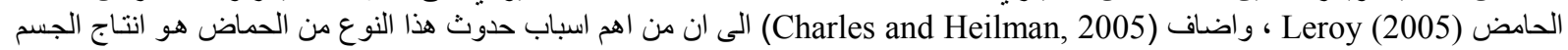

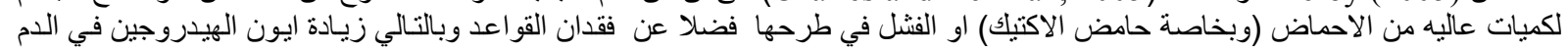

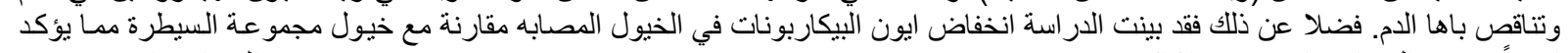

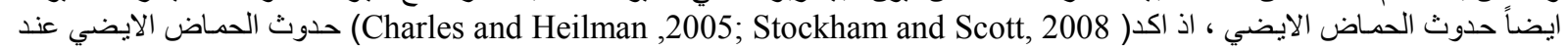

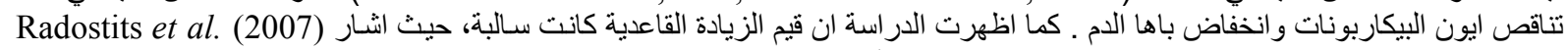

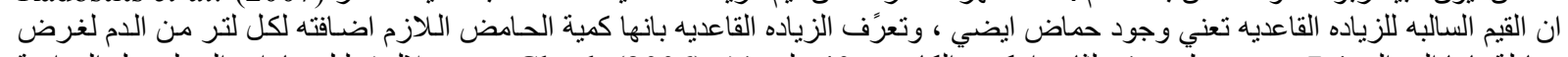

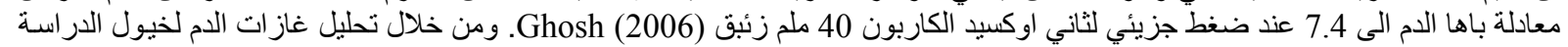

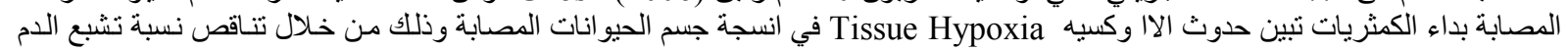

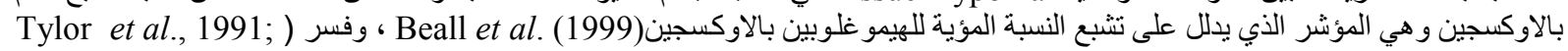
(Tylor, 1993

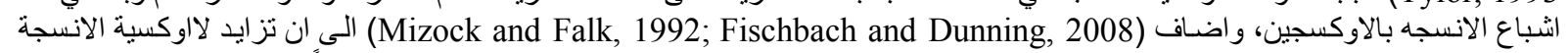

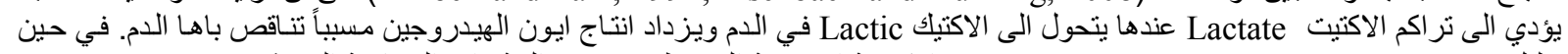

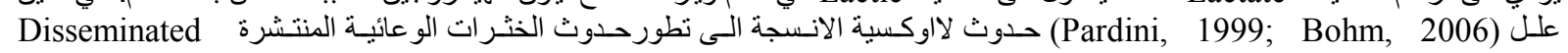
الاوكئ intravascular coagulopathy

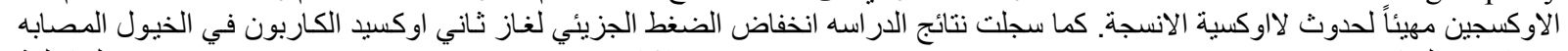

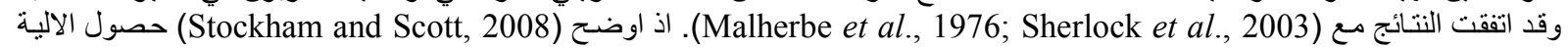

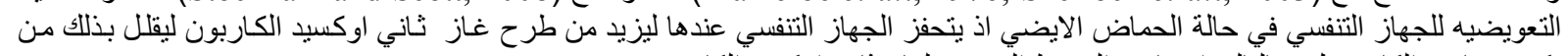
تكوين حاض الكاربونيك وبالتالي انخفاض الضغط الجزيئي لغاز ثاني اوكسيد الكاربون.

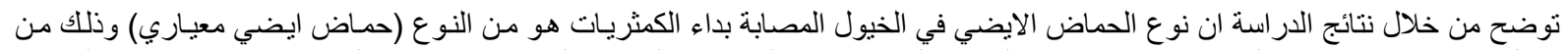

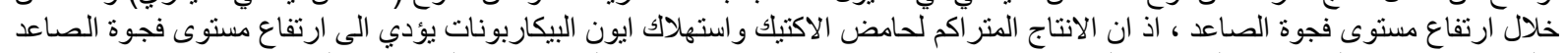

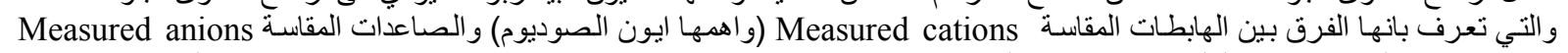

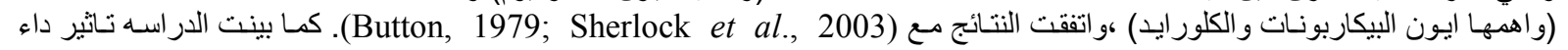

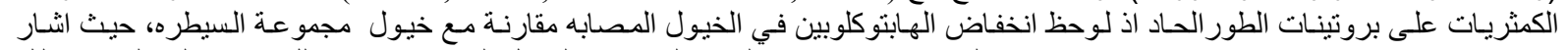

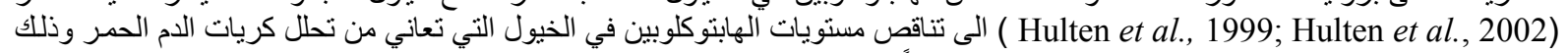

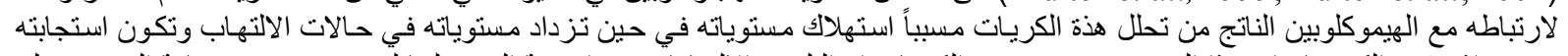

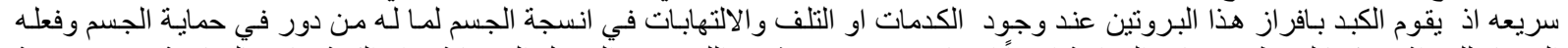

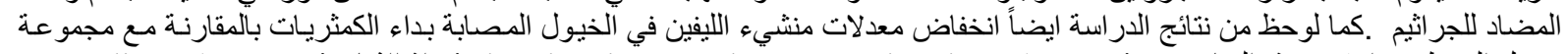

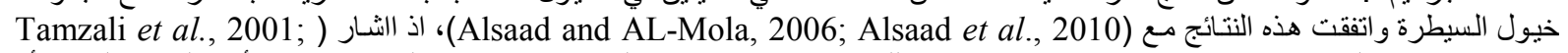
(Smith, 2004 بسبب استهاكه في عمليات التنجلط وحدوث الخثرات الو عائيد المنتشره.

\section{REFERENCES}

Alsaad, K.M.; Alsaad, E.A. and Al-Derawie, H.A. (2010): Clinical and Diagnostic Study of Equine Babesiosis In Drought Horses In Some Areas of Basrah Province. Res. J. Anim. Sci. 4(1)16-22.

Alsaad, K.M. and AL-Mola, G.M. (2006): Clinical and pathological study of equine babesiosis in drought horses in Mosul. Iraqi. J. Vet. Sci. 20(1): 89-101.

Alssad, K.M. (2009): Acute Babesiosis in foals. J. Anim. Vet. Adv. 8(12)2585-2589.

Beall, C.B.; ALmasy, L.A.; Blangero, J.; Williams-blangero, S. and Brittenham, G.M. (1999): Percent of Oxygen Saturation of Arterial Hemoglobin Among Bolivian Aymara at 3,900-4,000 m. Amer. J. Physic. Anthropol. 108:41-51

Bohm, M. (2006): Capillary and venous Babesia canis rossie parasitemia and their association with outcome of infection and circulatory compromise, Vet. Parasitol. 141: 18-24.

Button, C. (1979): Metabolic and electrolyte disturbances in acute canine babesiosis. JAVMA .175(5): 475-975.

Ceron, J.J.; Eckersall, P.D. and Subiela, S.M. (2005): Acute phase proteins in dogs and cats: current knowledge and future perspectives. Vet Clin Pathol. 34:85-99.

Charles, J.C. and Heilman, R.L. (2005): Metabolic acidosis .www.Turner- White.com.

Fischbach, F.T. and Dunning, M.B. (2008): Manual of Laboratory \& diagnostic testes. $8^{\text {th }}$.ed Saunder company. Philadelphia. pp.973.

Ghosh, A.K. (2006): Diagnosis acid base disorder. J. Asso. phys. 47: 29-33.

Gruys, E.M.J.M.; Touissant, W.J.M.; Landman, M.; Tivapasi, R.; Chamanza, L. and Van Veen. (1999): Infection, inflammation and stress inhibit growth. Mechanisms and non-specific assessment of the 
processes by acute phase proteins, In: T.Wensing (ed.), Production diseases in farm animals, 2, Ageningen Press. The Netherlands. pp. 72-87.

Gruys, E.; Obwolo, M.J. and Toussaint, M.J.M. (1994): Diagnostic Significance of the major acute phase proteins in veterinary clinical chemistry :a review. Vet. Bull. 64: 1009-1018.

Hiss, S.; Mielenz, M.; Bruckmaier, R.M. and Sauerwein, H. (2004): Haptoglobin Concentrations in Blood and Milk After Endotoxin Challenge and Quantification of Mammary Hp mRNA Expression. J. Dairy Sci. 87: 3778-3784.

Hulten, C.; Gronlund, U.; Hirvonen, J.; Tulamo, R-M.; Suominen, M.M.; Marhaug, G. and Forsberg, M. (2002): Dynamics in serum of the inflammatory markers serum amyloid A (SAA), haptoglobin,fibrinogen and alpha(2)-globulins during induced noninfectious arthritis in the horse. Equine Vet. J. 34: 699-704.

Hulten, C.; Sandgren, B.; Skjoldebrand, E.; Klingeborn, B.; Marhaug, G. and Forsberg, M. (1999): The Acute phase protein serum amyloid A (SAA) as an inflammatory marker in equine influenza virus infection, Acta. Vet. Scand. 40: 323-333.

Hunfeld, K.P.; Hildebrandt, A. and Gray, J.S. (2008): "Babesiosis: Recent insights into an ancient disease". Int. J. Parasitol. 38 (11): 1219-37.

Jain, N.C. (1993): Essentials of Veterinary Hematology. $1^{\text {st }}$ ed. Lea and Febiger. Philade-lephia. pp: 121-123.

Johnson, P.J. (1995): Electrolyte and acid-base disturbances in the horse. Vet. Clin. North Am. Equine Pract. 11(3): 491-514.

Katsuhiko, Y. (2002): Colorimetric determination of chloride in biological samples by using mercuric nitrate and phenylcarbazone. Bio. Tra.Ele.Res. 85(1): 87-94.

Leech, N.L.; Barrett, K.C. and Morgan, G.A. (2007): SPSS for intermediate statistics: use and interpretation. $1^{\text {st }}$ ed, Lawrence Erlbaum Asso. USA.pp: 20-51.

Leisewitz, A.L.; Jacobson, L.S.; De Morais, H.S. and Reyers, F. (2001): The mixed acid based disturbances of severe canine babesiosis. J. Vet. Intern. Med. 15: 445-452.

Leroy, B.E. (2005): Acid base balance, Clinical Pathology (VPAT 5250) notes, pp.1-21.

Lobetti, R.G.; Davir, E. and Pearson, J. (2002): Cardiac troponin in canine babesiosis. J. Vet. Intern. Med. 16 : 63-68.

Malherbe, W.D.; Immel man, A.; Haput, W.H. and Wazl, H.J. (1976): The Diagnosis and Treatment of acid base balance deranged dogs with Babesia canis. J. South Africa Vet. Asso. 47: 29-33.

Mark, K.K. (2010): Equine piroplasmosis in Greece. J. Vet. Parasitol. 169: 273-278.

Mizock, B.A. and Falk, J.L. (1992): Lactic acidosis in critical illness. Crit. Care Med. 20: 80-93.

Mohri, M. and Sardari, K. (2000): Clinical and laboratory findings in equine piroplasmosis. J. Equine. Vet. Sci. 20: 857-859.

Murtaugh, M.P.; Baarsch, M.J.; Zhou, Y.; Scamurra, R.W. and Lin, G. (1996): Inflammatory cytokines in animal health and disease. Vet. Immunol. Immunopathol. 54. 45-55.

Oladosa, L.A. and OllFeml, B.E. (1992): Hematology of experimental babesiosis \& enrlichiosis in steroid immunosuppresscal horse. J. Vet. Med. 39: 345-352.

Pardini, A. (1999): The pathology of canine babesiosis .pretoria: university of Pretoria.

Petersen, H.H.; Nielsen, J.P.; Helweg, P.M. and Heegard, K. (2004): Application of acute phase protein measurements in veterinary clinical chemistry. Vet. Res. 53. 163-187.

Radostitis, O.M.; Gay, C.C.; Blood, D.C. and Hinchliff, K.W.(2007): Veterinary Medicine. A text book of the diseases of cattle, sheep, goats and horses.10 ${ }^{\text {th }}$ ed, WB Saunders Co.pp: 1483-1498.

Salem, A.E.G.; El-Battawy, M.A.; El-Seify, M.; Salim, K. and Hosny, Z. (1986). Preliminary investigation on hematological and Bio-chemical picture of blood of premunized donkeys against Babesia equi. Assiut. Vet. Med. J. 16: 147-155.

Sherlock, M.; Healy, A.M. and Doherty, M.L. (2003): Acid base balance in field cases of bovine babesiosis. Vet. Rec. 152(22)687-688.

Shiroshita, Y.; Tanaka, R.; Shibazaki, A. and Yamane, Y. (1999): Accuracy of aPortable Blood Gas Analyzer Incorporating Optodes for Canine blood. J. Vet. Int. Med. 13(6)597-600.

Smith, B.P. (2004): Large animal internal medicine, $4^{\text {th }}$ ed., New York, Mosby. pp: 1144-1154.

Stockham, S.L. and Scott, A.M. (2008): Fundamentals of Veterinary Clinical Pathology. Iowa state press, pp. $383,385,393$.

Suffredini, A.F.; Fantuzzi, G.; Badolato, R.; Oppenheim, J.J. and O'Grady, N. (1999): New insights into the biology of the acute phase response, J. Clin. Immunol. 19: 203-214. 
Tamzali, Y.; Guelfi, J.F. and Braun, J.P. (2001): Plasma fibrinogen measurement in the horse : comparison of Millar's technique with a chronometric technique and the QBC Vet. AutoreaderTM. Res. Vet. Sci. 71(3): 213-217.

Tylor, J.H. (1993): The effect of Babesia canis induced hemolysis on the canine hemoglobin oxygen dissociation curve. J. South Africa Vet. Asso. 64: 141-143.

Tylor, J.H.; Guthrie, A.J. and Leswitz, A. (1991): The effect of endogenously produced carbon monoxide on oxygen status of dogs infected with Babesia canis. J. South Africa Vet. Asso. 104: 27-41.

Vannier, E. and Krause, P.J. (2009): Update on babesiosis. Inter. Persp. Inf. Dis. 10: 1-9.

Welzl, C. (2001): Systemic inflammatory response syndrome and multiple organ damage, dysfunction in complicated canine babesiosis. J. South Africa Vet. Asso. 72: 158-162.

Zambelli, A.B. (2007): Comparation of the effect of packed red cell transfusion and oxyglobin in canine babesiosis. MMed. Vet. 1-127.

Zobba, R.A.; Mauro, A.B.; Serena, N.B.; Bernardo, C.C.; Laura, M.D.; Raffaella, C.E. and Maria Luisa, P.P. (2008): Clinical and Laboratory Findings in Equine Piroplasmosis. J. Equine. Vet. Sci. 28(5): 308-310. 